International Journal of

Advanced Science and Convergence

\title{
A Study on the Prospect of Using IoMT for Disease Prevention and Management
}

\author{
Sungho Sim ${ }^{1}$ \\ ${ }^{1}$ College of General Education, Semyung University, Jecheon, 27136, Korea
}

\begin{abstract}
Background/objectives: With the development of IT technology and interdisciplinary convergence technology, interest in medical services, healthcare, bioinformatics, and public management is increasing. It is changing from passive disease management to active disease management using IoT devices. Methods/Statistical analysis: Due to the paradigm shift in the medical and health industries due to the Fourth Industrial Revolution, interest in disease prevention including disease management is increasing. Research on the Internet of Medical Things is being actively conducted as a method for such disease prevention and management services. Therefore, in this paper, we will examine medical ICT devices, healthcare devices, and IoMT in the IoT environment, and propose an efficient use method of IoMT for disease prevention and management and a prospect for this. Findings: In order to smoothly operate disease prevention and management based on the IoMT environment, problems such as expansion of ICT infrastructure, development of the system, and training of experts are expected to be solved. Improvements/Applications: In addition, in the use of IoMT, reorganization of policies and legal systems, improvement of security technology and awareness of personal information, and user consent for sensitive information are tasks to be resolved.
\end{abstract}

\section{Index Terms}

Healthcare, ICT, IoT, IoMT, Disease management.

\author{
Corresponding author : S. H. Sim \\ shshim@semyung.ac.kr \\ - Manuscript received July 6, 2020. \\ - Revised August 5, 2020 ; Accepted September 21, 2020. \\ - Date of publication September 30, 2020.
}

(C) The Academic Society of Convergence Science Inc.

2619-8150 ( 2020 IJASC. Personal use is permitted, but republication/redistribution requires IJASC permission. 


\section{INTRODUCTION}

The IoT environment-based medical industry and IT convergence service-related fields are steadily growing and increasing. Health care IoT is a field that deals with information, devices, systems, and platforms related to personal health and medical care and provides personal health care services. The main goal of healthcare IoT is to monitor the user's symptom and treatment management status in an always-connected environment so that there is no gap in disease management. IoT is connected through a network such as devices, sensors, and actuators [1-3]. Wearable is a IoT device to manage the user's health and safety. Wearable devices support sensor and built-in technology for data communication that can interact with the environment, and can continuously monitor user activities through physical networks [4,5]. Real-time data collection through monitoring can generate information on personal health. The relationship between health information can be used for management of user health and disease prevention by analyzing and inferring the complex meaning of one information using artificial intelligence such as statistical techniques and machine learning. Research for user data processing in IoT environments is being conducted [6,7]. IoMT is emerging as a service that can provide customized health management by constantly monitoring the patient's symptoms and treatment management status through various data such as user's biometric information, medical use information, disease information, and genome information. In this paper, the introduction describes the research background and the necessity of IoMT. Next, in Chapter 2, IoMT overview and processing technology are reviewed, and in Chapter 3, the relationship between IoMT and IoT is examined, and in Chapter 4, use cases, prospects, and implications for IoMT-based services are described, and it is composed of conclusions.

\section{RESEARCH BACKGROUND}

\section{A. Internet of Medical internet Overview}

IoMT is a health care service and IT convergence service that collects user monitoring information through the IoMT device for user health and disease information. Through the establishment of the IoMT environment, customized case management and disease prevention services are provided to users regardless of time and place. In order to represent the infrastructure connected with medical devices and software applications that can communicate with various medical IT systems, the concept of Internet of Medical Things is established and defined [8]. The core of health care IoT is to prevent gaps by monitoring user symptoms and treatment management status [9]. Beyond the level of integrating and analyzing user monitoring information, it is being used as a means to improve the quality of life of patients and to realize patientcentered service values. In addition, IoMT can lower social and economic costs through business model change by integrating health care and IT. It is expected that demand will increase through solutions to various problems caused by the aging of the population and to supply digital healthcare policies. IoMT is based on On-Demand Healthcare service, and provides the service as soon as the user wants. In the United States, commercialization is taking place as an alternative to reduce the cost of medical services. In addition, the market maturity is high due to the high demand for on-demand healthcare combined with telemedicine. IoMT technology presents the user's disease prevention and management service model by applying the user's condition measurement, medical asset control, and health data integration. These Health care service models are expected to promote medical service model changes in accordance with demands of users [10]. In addition, the expansion of the cloud-based IoMT platform can create the effect of pioneering a new market in the healthcare field and the development of new services through strengthening strategic alliances between hardware and software suppliers and analysis software suppliers. The following figure 1 shows the IoMT $[11,12]$.

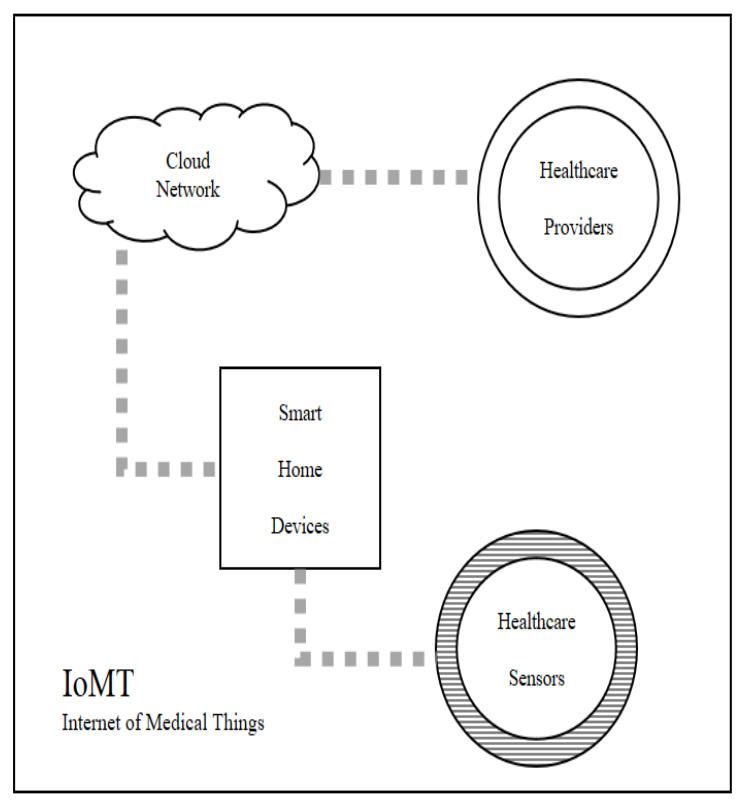

Fig. 1. IoMT(Inter of Medical Things) 


\section{B. IoMT processing technology}

Disease prevention and management using wearable devices includes an area that can perform computing functions by collecting user information using a device. Based on the classification, it is divided into portable type, adhesive type, and transplantation / eating type, and the range of utilization changes according to the user. For individuals, it is used in the Activity Tracker, which collects and analyzes user monitoring information in the field of disease prevention and management services[13]. A sensor is a device that detects information such as temperature, environment, biological signals, and movement from an object and changes them into signals. Sensors are developing into smart sensors with standardized interfaces and information processing capabilities in order to improve application characteristics. Multi-sensor technology can extract intelligent and high-level information. Network infrastructure technology refers to all wired and wireless networks that can connect humans to objects, objects to objects such as WPAN, LTE/4G/5G, bluetooth, ethenet, and microware. Interface technology plays a role in interworking with application services that perform specific functions in the three major components of IoT: humans, objects, and services. That is, it serves as an interface to provide services such as sensing, extracting/analyzing, storing, and determining information, context recognition, recognition, authentication/authorization, web service technology, and middleware technology. IoT and disease prevention and management services are convergence industries that can provide usercentered services. Figure 2 shows the function of the Wearavle device [14].

\begin{tabular}{|c|l|}
\hline Function & \multicolumn{1}{|c|}{ Contents } \\
\hline $\begin{array}{c}\text { Constant } \\
\text { castle }\end{array}$ & $\begin{array}{l}\text { - There exists a channel(Internet, } \\
\text { Bluetooth, etc.) that can communicate } \\
\text { with real time computer }\end{array}$ \\
\hline Convenience & $\begin{array}{l}\text { - Provide sense of identity and } \\
\text { integration that users can use naturally } \\
\text { and conveniently }\end{array}$ \\
\hline Fit & $\begin{array}{l}\text { - When wearing or attaching the user's } \\
\text { body, provides lightness and naturalness }\end{array}$ \\
\hline Stability & $\begin{array}{l}\text { - Minimize fatigue and skin troubles } \\
\text { caused by wearing or attaching for a } \\
\text { long time, ensuring safety against power } \\
\text { and electromagnetic waves }\end{array}$ \\
\hline Sociability & $\begin{array}{l}\text { - Protection of privacy exposure by } \\
\text { recording/recording and hacking }\end{array}$ \\
\hline
\end{tabular}

Fig. 2. Function of Wearable Device .

\section{ICT IN THE MEDICAL INDUSTRY}

\section{A. Combination of IoT and medical services in the health industry}

In the health care industry, many studies are currently being conducted on disease prevention and management. With the spread of cloud-based ICT platforms, new convergence services such as communication service providers, hardware suppliers, and analysis software suppliers are supported. This convergence service continues to grow the ICT device connection and service support platform business participating in the health care industry. In addition, the fusion of IoMT and mobile technology is driving the growth of smart mobile healthcare, which is an IoT-driven application field. Smart mobile healthcare provides daily and comprehensive healthcare services using devices such as user monitoring devices, mobile devices, personal information terminals, and sensors. Wearable based services can receive medical service support automatically through cloud computing and analysis software using data collected through wearable devices. Bio signals and health information are collected in real time from sensors attached to the user's body through the connection of wearable devices. As the demand for smart warable devices increases, the demand for warable based IoMT services is increasing.

\section{B. Disease prevention and management service using IoMT}

With the development of IoT technology, the medical field using IoT technology is changing to support new services. In particular, IoT sensors used in medical services can be attached and detached from the user's body, and user information can be collected in real time regardless of time and place. IoT-based medical service supports a new type of service by integrating various element technologies, so it is necessary to accurately process the collection and analysis of information generated by IoT devices within a minimum amount of time. It delivers a large amount of information collected through IoT devices to the disease control center using wired or wireless devices. The Centers for Disease Control and Prevention provides IoMT-based services so that disease prevention and management services can be effectively performed by checking collected various user information. The disease prevention and management support service analyzes and selects monitoring information to provide support services by constructing probability information according to attribute information in order to accurately and quickly find information for treatment and management among users' information. IoMT environment-based disease prevention and 
management service improves the speed of collecting and analyzing monitoring information by hierarchically managing similar information according to the type, function, and characteristics of monitoring information collected through ICT and IoMT devices. The hierarchically organized information allows algorithms to work together to apply in large scale disease prevention and management service environments. Through clustering similar information among hierarchically managed information by combining various attribute information, it is possible to provide accurate and customized support for medical service prescriptions and management plans for disease information. Figure 3 shows the disease prevention and management service process.

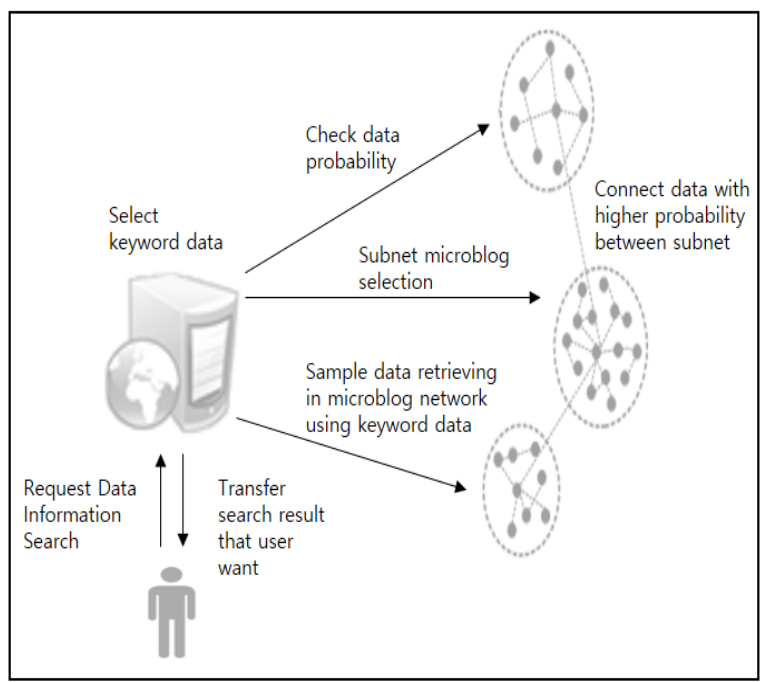

Fig. 3. Overall process of Scheme .

\section{IOMT BASED DISEASE PREVENTION AND MANAGEMENT}

\section{A. IoMT Service}

Medical IT Service Company in the United States is supporting services by innovating a blood sampling service process model for medical diagnosis. Service Company provides an on-demand service for the blood collection process performed at the user's medical institution. The mobile based ondemand service method handles the process from blood collection to transportation and management, and notification of test results. Such a service can save users' time and cost. It provides convenience to users and medical staff by streamlining communication between medical staff and blood collection room. The service company platform network makes an appointment with the person who can immediately perform the request among registered blood collection personnel and laboratories, and determines the place to support the service. This service operation model supports all participants to convert work efficiency and convenience. Figure 4 shows the service company model [15].

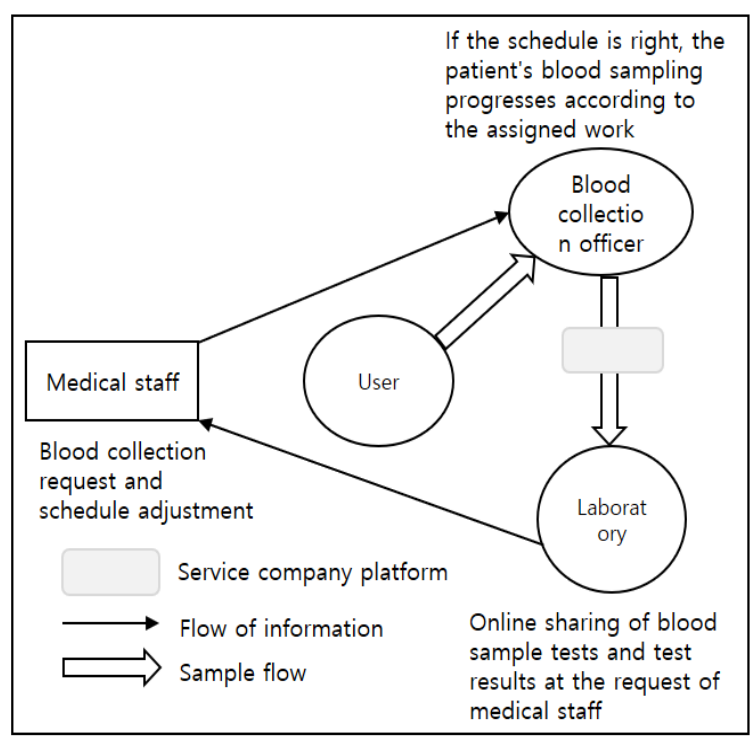

Fig. 4. service operation model.

\section{B. Outlook}

The need for IoMT is increasing due to the increasing social and economic costs associated with health care and disease due to an aging population. In addition, IoT based solutions are expected to expand due to the spread of the IoT environment and the spread of ICT devices. Currently, IoMT is being commercialized mainly in North America and Europe, and the market is expanding mainly in developed countries. Adoption of telemedicine services and the development of IoMT devices that can be applied to the user's body are actively progressing. An environment that can provide customized medical services to users such as sensor chips that can be implanted on the user's body and wearable devices that can be attached to the user is being built. The ICT industry is also changing from passive health management to a fusion model capable of active health management and disease prevention through the development of sustainable business models. With the introduction of IoMT, more services can be supported through continuous convergence of various related technologies and expertise. 


\section{Suggestion}

Korea's IT infrastructure construction is excellent. However, there are a lot of lack of medical support services in the IoT environment. In order to smoothly operate IoT-based medical services, it is necessary to expand IoMT, develop analysis and application software, and nurture experts. In the initial stage of IT-based disease prevention, the health industry should consider expanding services and establishing a support system through active use of IoMT. It is possible to establish guidelines for disease prevention services by intensive, linking, and selection of requirements and health care information requested by users in one place. Considering that the software part suitable for the user's environment is weak, it is also necessary to prepare a system for securing experts in health industry data in order to strengthen long-term industrial competitiveness. In addition, there are a number of tasks to be solved, such as improvement of insufficient legal system in the use of IoMT, improvement of security technology and awareness of monitoring information, and concentration of data. As it is a newly emerging field, it is necessary to improve the institutional and legal system that can utilize innovation capabilities while minimizing expected side effects through sufficient case benchmarking. In order to prevent the indiscriminate use of sensitive information generated in the IoMT environment, guidelines for the purpose and scope of data collection and utilization, procedures and validity must be established. The social side effects expected from the monopoly of sensitive information should be reviewed and discussed on supplementation.

\section{Conclusion}

With the development of IT technology and convergence technology, interest in medical service, healthcare, bioinformatics, and public management is increasing. Although it supports healthcare services using medical ICT, it is insufficient to apply to the prevention and management of diseases. New medical service support is possible through the convergence of medical industry and IT. IoMT environment-based disease prevention and management services can support disease management and prevention services required by health care. IT based medical service support is still in its infancy. However, the advantages of excellent IT infrastructure and economics mean that it is well worth investing in disease prevention and management services. In this paper, we investigated medical ICT devices, healthcare devices, wearable, and IoMT in the IoT environment, and investigated and analyzed IoT based medical service use cases, and presented effective ways to use IoMT for disease prevention and management and its prospects. In the initial stage of disease prevention and management services based on the IoMT environment, it is necessary to establish an integrated governance system to lead to the establishment of an active IoMT environment and creation of added value in the medical health industry. In addition, there is a need to prepare a system for securing experts in health care data. As it is a new convergence service field, it is required to improve the system and the legal system to utilize the innovation capabilities, and to sufficiently review and secure the side effects of the use of sensitive information.

\section{REFERENCES}

[1] Ray, S., Park, J. S., \& Bhunia, S. (2016). Wearables, Implants, and Internet of Things: The Technology Needs in the Evolving Landscape. IEEE Transactions on Multi-Scale Computing Systems, 2(2), 123-128. DOI : 10.1109/TMSCS.2016.2553026

[2] Yoo, J. H. (2016). A Study on Implementation of System Improvement for Medical Information Processing. Journal of Digital Convergence, 14(11), 283-288.

DOI : 10.14400/JDC.2016.14.11.283

[3] Song, M. H. (2020). Vocabulary model and User context-based Speech Recognition assistant System for the elderly living alone. International Journal of Advanced Science and Conbergence, 2(2), 9-14.

[4] Wei, J. (2014). How Wearables Intersect with the cloud and the Internet of Things: Considerations for the developers of wearables. IEEE Consum. Electron, 3, 53-56.

DOI : 10.1109/MCE.2014.2317895

[5] Haghi, M., Thurow, k. \& Stoll, R. (2017). Wearable devices in Medical Internet of things: Scientific Research and Commercially Available Devices. Healthc. Inform. Res. 23, 4-15. DOI : 10.4258/hir.2017.23.1.4

[6] Manyika, J. (2011). Big data: The next frontier for innovation, competition, and productivity. Http://www.mckinsey.

com/insights/MGI/Research/Technology_and_Innova tion/Big_data_The_next_frontier_for_innovation.

[7] World Economic forum. (2012). Big data, big impact:New possibilities for international development. https://www.weforum.org/reports/bigdata-big-impact-new-possibilities-internationaldevelopment.

[8] Tim. S. (2016). New medical device technology set to benefit patients. Digital Journal.

http://www.digitaljournal.com/tech-andscience/technology/five-important-medicaltechnology-trends/article/478161

[9] Uttam. K. (2017). Evolution of IoT playing a crucial role in the healthcare sector. Data Quest. https://www.dqindia.com/evolution-of-iot-playing-acrucial-role-in-the-healthcare-sector/ 
[10] Frost \& Sullivan. (2017). Internet of Medical Things (IoMT) Revolutionizing Healthcare.

[11] Klonoff DC. (2017). Fog computing and edge computing architectures for processing data from diaberes devices connected to the medical internet on things. J Diaberes Sci Technol, 11, 642-652.

[12] Rami, B., Bijan, N. \& David G. A. (2018). Health Sensors, Smart Home Devices, and the Internet ofMedical Things: An Opportunity for Dramatic Improvement in Care for the Lower Extremity complications of Diabetes. Journal of Diabetes Sciene Science and Technology, 12(3), 577-586.

[13] Kim, J. H. (2015). A Study Health Care Smart system based on IoT. Incheon National University.

[14] Jeong, Y. S. (2017). Data Storage and Security Model for Mobile Healthcare Service based on IoT. Journal of Digital Convergence, 15(3), 187-193. DOI : 10.14400/JDC.2017.15.3.187

[15] Kim. S. B. (2017). Health Industry 4th Industrial Revolution Series: Global Medical Internet of Things(Internet of Medical Things, IoMT) Market Trend. KHIDI Brief. 205, 1-16. 